\title{
Pre-Design Methodology of Voltage Inverters using a Gradient-based Optimization Algorithm
}

\author{
Adrien Voldoire, Jean-Luc Schanen, Senior Member, IEEE, Jean-Paul Ferrieux, Benoit Sarrazin, \\ Cyrille Gautier, Member IEEE
}

\begin{abstract}
This article presents an optimization process applied to the sizing of high power silicon carbide inverters. The case study is the design of an aeronautical converter. A gradient-based optimization method is chosen for its ability to explore a wide solution space with a large amount of constraints, what is the case during the pre-design phase. Many topologies are selected and associated models are developed. Among all these models, active and passive component design, and spectral analysis of voltages and currents, are presented. Optimization results, with the goal of minimizing the global mass of the converter, are provided. The different topologies are compared to find the best one for the selected case study. The relevance of the optimization result is evaluated with the study of the optimality of the solution. Finally, experimental results are compared to the optimization ones, which validates the good accuracy of analytical models.
\end{abstract}

Index Terms - Optimization, Design Automation, Voltage Source Inverter, SiC, Interleaving

\section{INTRODUCTION}

$\mathrm{C}$ URRENT aircraft systems use multiple energy vectors for non-propulsive actuators: mechanical, electrical, pneumatic and hydraulic. Pneumatic and hydraulic systems are heavier and more costly in terms of maintenance compared to electrical systems. Considering also the growth of air traffic, and the necessary requirement of reduced fuel consumption, the concept of More Electrical Aircraft (MEA) appeared few years ago [1]. The goal is to improve the overall efficiency of aircraft systems by increasing the use of electrical power on board, instead of hydraulic or pneumatic. This increase in electrical power requires thinking about a new organization of the embedded aircraft grid and the associated converters. Power converters have to be functional, with the lowest possible mass and with the respect of the standards, as DO160 in the civil domain.

Building a converter with a minimal mass and respecting several constraints is a tedious task with a traditional sizing approach, usually involving designing components one by one. Indeed, multiple compromises appear, for example on the choice of the switching frequency, which has an opposite impact on the heatsink sizing and on the filter design. This is the reason why an optimization strategy is necessary, especially

Manuscript submitted on June $20^{\text {th }}, 2020$, revised on October $22^{\text {nd }}, 2020$, revised on January $14^{\text {th }}, 2021$, revised on May $19^{\text {th }}, 2021$, accepted on June $15^{\text {th }}$, 2021 .

This work was partially supported by Safran Tech. in the early steps of the design, where huge degrees of freedom are open, and no one can fully investigate each of them.

Two major families of design tools can be found in the literature, illustrated by Fig. 1. Stochastic methods can sweep many points of the solution space using for instance evolutionary algorithms. This kind of tool enables a precise design of a converter but needs some time for the solving. Multiple examples of stochastic optimization of power converters are available. The structure of the stochastic algorithm is modified in [2] to reduce the number of combinations by $99 \%$ in the case of a DC-AC converter. Different structures and filters are compared for grid-tied inverters in [3] without a global approach: components are designed in separated loops. A design space analysis is performed in [4] to understand which filter structure respect the constraints. Multi-objective optimization is carried out in [5] in order to increase the reliability of solar inverters. Authors from [6] use two concepts to improve NSGA-II algorithm in order to achieve an accurate multi-objective optimization.
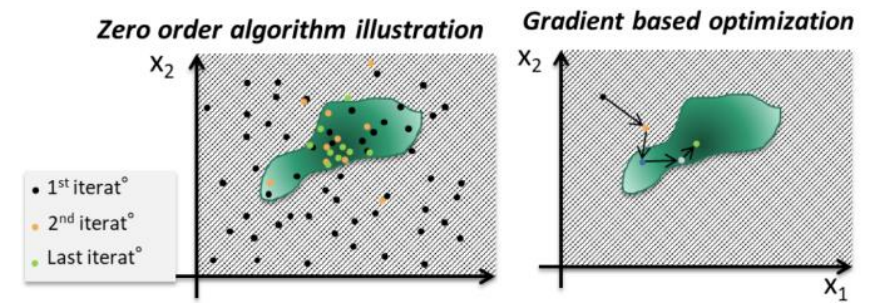

Fig. 1. Comparison between stochastic and gradient-based method

On the other hand, deterministic algorithms make it possible to converge quickly to the optimal point. For example, multilevel DC/DC converters are designed in few seconds in [7] using a geometric programming algorithm.

Solving time is a key issue during the predesign step of a power converter, where the space of solutions is still wide, and several possibilities are offered to the designer. That is one of the reasons to choose this kind of methods. However, gradientbased methods need computing the gradients of the equations, meaning that these ones need to be differentiable.

In other words, they require using continuous and differentiable equations, therefore defining the notion of

A. Voldoire, J.L Schanen, J.P Ferrieux, and B. Sarrazin are with Univ. Grenoble Alpes, 38000 Grenoble, France (e-mail: jeanluc.schanen@g2elab.genoble-inp.fr).

C.Gautier is with Safran Tech, 78114 Magny-les-Hameaux, France (cyrille.gautier@safrangroup.com). 
"imaginary world" [8]. It is a relaxation of the real-world, where the components are not always available in manufacturer catalogues, and where some discrete parameters can have noninteger values (as the number of turns for winding, or the interleaving level). The optimum point reached in this world usually gives sufficient data for the engineer in the pre-design step of a project, a period where the highest impact decisions are taken. With a gradient-based optimization approach, it is also possible to analyze the impact of the constraints on the characteristics of the converter, what is very often a key point during the predesign steps, where requirements are not fully fixed.

An important aspect of an optimization problem is the way it is set up, i.e. how design variables and constraints are chosen. In this paper, physical and technological variables are chosen as inputs. This is particularly relevant considering magnetic components (inductors and magnetic couplers) are not standard devices at power levels used in the targeted applications. They are designed by choosing a magnetic material, a core size, a specific conductor (plain or Litz wire) and turn numbers. This choice of description, based on the technology, is representative of the final design, accounts for components constraint, and offers more degrees of freedom than choosing devices in a catalogue as in [7]. Furthermore, as stated before, such standardization does not exist, except for low power.

As a result, the optimization variables are not all defined in the electrical world: usually, the inductance is first defined, according to the various converter electrical requirements, and then the component is optimized in a second step, to minimize its weight or volume, meeting internal constraints as maximum losses or temperature. This paper directly solves this issue by including the design of the magnetic devices with the overall system. This can improve results compared to a sequential design approach, as a global optimum may not necessarily be a superposition of the optima of the individual.

Exploring a large solution space requires the models to be valid over an equally large range. Not only because the optimal solution may be found in an "unusual" location, but also because during the optimization process, the objective function and the constraints may be evaluated at these points, and it is necessary that the information from the models are still relevant. Therefore, care has to be taken to build models with wide validity range. For instance, it is quite common to consider the output current of a PWM inverter as a perfect sinewave, i.e. neglecting the current ripple. This assumption is perfectly valid for most of the conventional design. However, due to the aforementioned reason, the proposed approach uses a complete model, considering the AC current ripple in the computation of the RMS current of the DC bus capacitor [16] (Section II-C).

Finally, all converters requirements are expressed as design constraints. For power quality for instance, a global THD (Total Harmonic Distortion) and individual harmonic amplitude can be requested. This necessitates some specific model formulations, expressed in the frequency domain.

The choice of such level of details in the modelling approach must be compatible with the differentiability requirement expressed earlier, but may be not compatible with a posynomial formulation as the one used in [7]. This is a trade-off: more freedom to formulate the model at the cost of performance, including computation speed and convergence, as illustrated in Fig. 2. However, it will be shown in this paper that the chosen optimization strategy allows reasonable computation time and good convergence.

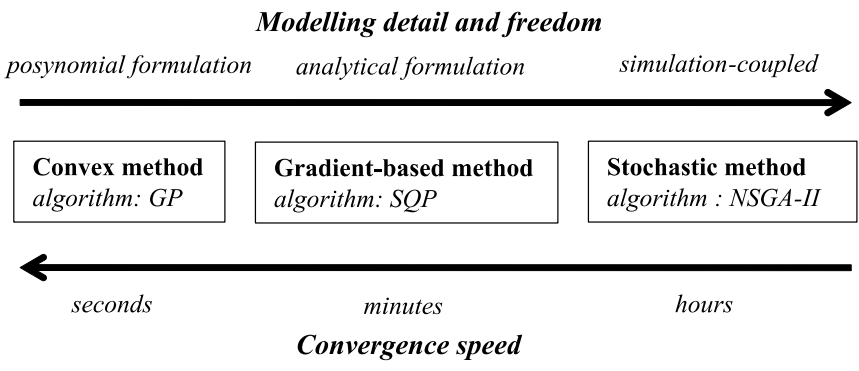

Fig. 2. Compromise between modelling effort and convergence speed for different algorithms

Relaxation of discrete parameters to allow differentiability will be described, and it will be shown that the number of variables and constraints can be very large (more than 1000), and that all interactions between parameters can be considered. Due to this high number of equations composing the full model, the manual computation of the gradients would be tedious, therefore a specific design environment has been used [26], which automatically computes the Jacobian matrix.

To illustrate the features of the proposed methodology, a high power inverter $(10 \mathrm{kVA}<\mathrm{S}<100 \mathrm{kVA})$ with output sinus filter for aircraft applications has been considered. As explained before and in comparison to previous work dealing with predesign strategies [7], the method was applied on a fully industrial application, involving power quality criteria on both input and output sides. In such high power inverter applications, inductors (not standard devices) are clearly a key component. Therefore, a particular focus has been made on a detailed description of its behavior, including saturation effects.

The output variable to minimize (objective function) is the total mass. As the global efficiency is also important, multiple optimizations are carried out for different efficiency constraints, which allowed a Pareto front to be drawn. The models implemented in the algorithm are partially described in the following section.

\section{ANALYTICAL MODELS DEDICATED TO INVERTERS}

As the goal of this work is to introduce a design methodology rather than developing models, this section will be kept short. A detailed bibliography about analytical models in power electronics is given in [9].

Table I and II summarize the main input parameters and constraints of the study. These latter are either defined at component level or at converter level. The semiconductor maximum temperature and the maximum RMS current in the capacitors must stay below the manufacturer requirements. Regarding inductors, to avoid the use of a complex thermal model, a maximum losses density has been defined. At converter level, power quality is imposed with several 
constraints: on the DC side, voltage and current ripples are imposed, whereas on the AC side, the voltage THD (Total Harmonic Distortion) is limited to $3 \%$, and the individual amplitude of each harmonic must stay below $2 \%$ of the fundamental voltage.

TABLE I

MAIN INPUT PARAMETERS

\begin{tabular}{llll}
\hline \hline \multicolumn{1}{c}{ Symbol } & \multicolumn{1}{c}{ Quantity } & \multicolumn{1}{c}{ Unit } & \multicolumn{1}{c}{ Value } \\
\hline $\mathrm{S}_{\text {load }}$ & Load power & VA & variable \\
$\mathrm{PF}$ & Load power factor & - & 0.9 \\
$\mathrm{~V}_{\mathrm{DC}}$ & DC voltage & $\mathrm{V}$ & 540 \\
$\mathrm{~V}_{\mathrm{AC}}$ & AC phase neutral & $\mathrm{V}$ & 115 \\
& RMS voltage & & \\
$\mathrm{F}_{\text {grid }}$ & Grid frequency & $\mathrm{Hz}$ & 400 \\
$\mathrm{~F}_{\mathrm{sw}}$ & Switching frequency & $\mathrm{Hz}$ & variable \\
$\mathrm{N}_{\text {interleaved }}$ & Interleaving level & - & variable \\
\hline \hline
\end{tabular}

TABLE II

MAIN CONSTRAINTS

\begin{tabular}{llll}
\hline \hline \multicolumn{1}{c}{ Symbol } & \multicolumn{1}{c}{ Quantity } & \multicolumn{1}{c}{ Unit } & \multicolumn{1}{c}{ Value } \\
\hline $\mathrm{T}_{\mathrm{J}}$ & Junction Temperature & ${ }^{\circ} \mathrm{C}$ & $<150$ \\
$\mathrm{THD}$ & AC Voltage THD & $\%$ & $<3$ \\
$\mathrm{H} \%$ & Individual harmonic & $\%$ & $<2$ \\
& limitation & & \\
$\Delta \mathrm{V}_{\mathrm{DC}}$ & DC voltage ripple & $\%$ & $<1$ \\
$\Delta \mathrm{I}_{\mathrm{DC}}$ & DC current ripple & $\%$ & $<5$ \\
$\mathrm{P}_{\text {tot vol }}$ & $\begin{array}{l}\text { Inductor volumetric } \\
\text { losses }\end{array}$ & $\mathrm{mW} / \mathrm{cm}^{3}$ & $<400$ \\
$\mathrm{I}_{\text {RMS C AC }}$ & $\begin{array}{l}\text { AC capacitor RMS } \\
\text { current }\end{array}$ & $\mathrm{A}$ & $<\mathrm{I}_{\text {nominal capacitor }}$ \\
$\mathrm{I}_{\text {RMS C DC }}$ & $\begin{array}{l}\text { DC capacitor RMS } \\
\text { current }\end{array}$ & $\mathrm{A}$ & $<\mathrm{I}_{\text {nominal capacitor }}$ \\
$\eta$ & Efficiency & $\%$ & Pareto \\
\hline \hline
\end{tabular}

The converter mass is the sum of the mass of the following components:

- power modules, with the mass provided by datasheets

- magnetic components, which are defined with geometric parameters (core diameter, core height, wiring and number of turn) and material density.

- capacitors, with mass interpolation from the datasheets, function of the capacity value.

- thermal exchanger, as explained in section II.B.

The last paragraphs present how to adapt the models to be compliant with the algorithm.

\section{A. Studied topologies}

Many topologies can be selected to achieve the inverter function. The basic structure is the 2-level 3-phase voltage inverter. Fig. 3 shows this topology with LC filters on the DC and $\mathrm{AC}$ sides.

Other structures can be considered in order to find the best design, including for example multilevel or interleaved inverters. Thus, this paper focuses on interleaving $N_{\text {interleaved }}$ inverters, with $N_{\text {interleaved }}$ AC inductor per phase (Fig. 4). Interleaving inverters decreases the current-related constraints on the passive and active components.

Another possible solution consists in coupling the AC inductors, either on one core for a monolithic structure, or on multiple cores for a cyclic cascade structure (Fig. 5) [10]. The cyclic cascade structure, and particularly the design of magnetic couplers, will be developed and optimized in the following paragraphs.

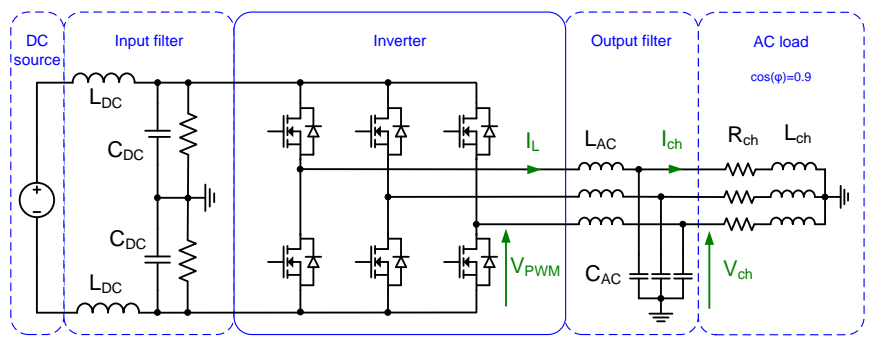

Fig. 3. 2-level 3-phase voltage inverter

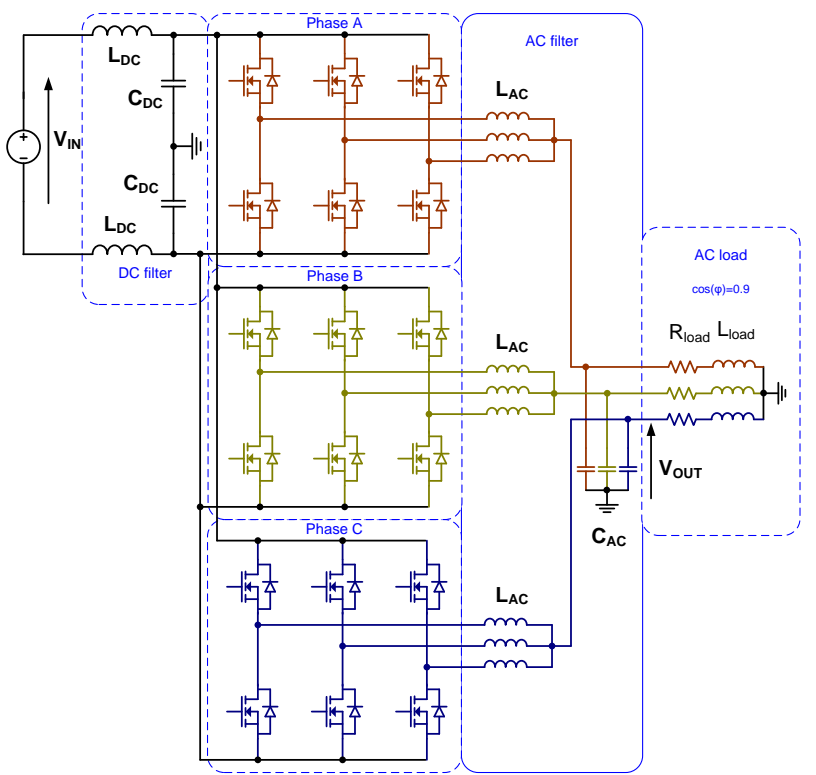

Fig. 4. 3-Phase inverter with 3 -interleaving non-coupled levels

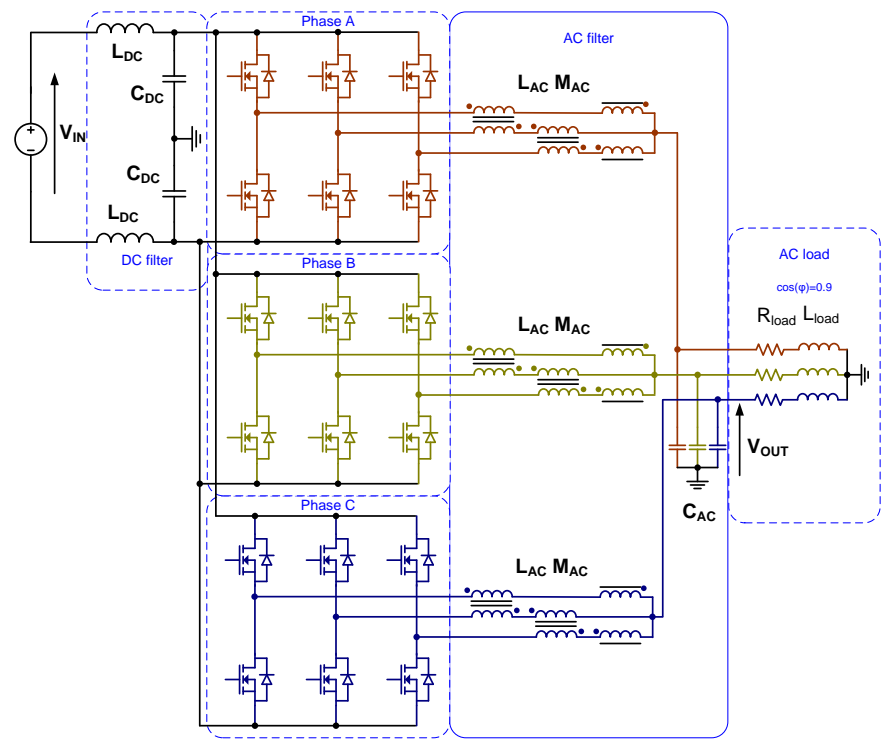

Fig. 5. 3-Phase inverter with 3 -interleaving coupled levels

The increase of the voltage levels, more and more considered for high power aircraft systems, leads the designer to think about multilevel structures. This topic will not be addressed in this study. 


\section{B. Semi-conductor design}

For this application, the chosen components are silicon carbide $(\mathrm{SiC})$ MOSFETs and Schottky diode, with a nominal voltage of $1200 \mathrm{~V}$. Phase-leg modules [11] are used. The computation of losses takes into account MOSFET switching losses, MOSFET and diode conduction losses, including the reverse conduction effect and the extra losses due to dead time [12], equal to 800ns. Conduction loss models require writing the RMS and average current in each active component in an analytical way.

The choice of the current rating for semi-conductors is based on the principle of a continuous variation of die area, which is a good illustration of the real world problem. Under the selected specifications (voltage and current) and for a given packaging, there exists an optimal die area to achieve minimum losses, illustrated on Fig. 6. This optimal area is a compromise between conduction losses, which tend towards a larger die, and switching losses, which increase for a very large chip because of the energy required to charge the Coss capacitor.

The optimal area depends also on the switching frequency, which has a significant impact on the other components of the converter. This is why the local optimization of the semiconductor as shown in Fig. 6 is just an illustration. All design formulas are integrated in the global converter design. In addition, as other constraints are interacting with this model, as the junction temperature, the optimal die area may not be the same as the one found by this local optimization: this justifies using a global approach.

Finally, the heat exchanger mass is computed using a rough evaluation of $1.5 \mathrm{~kg}$ per $\mathrm{kW}$ of losses, which is a reasonable assumption in aircraft systems [13].

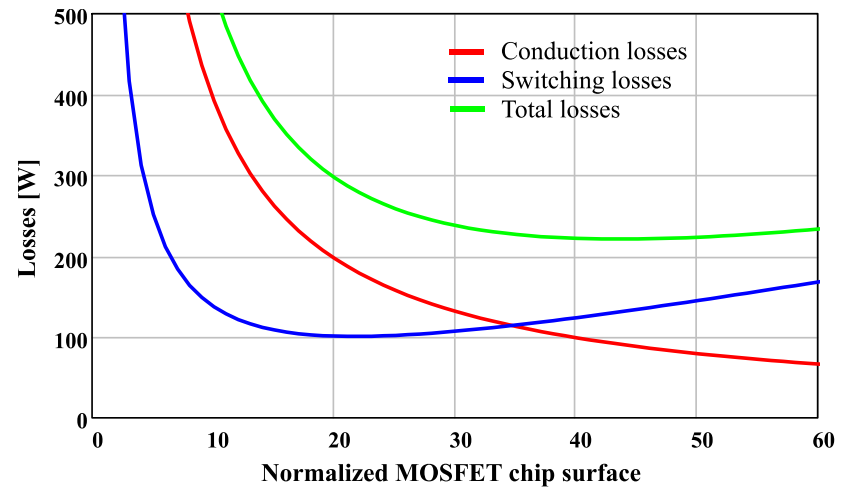

Fig. 6. Losses function of a normalized area of chip $\left(\mathrm{F}_{\mathrm{Sw}}=40 \mathrm{kHz}\right)$

\section{Spectral analysis}

Harmonic analysis of DC and AC voltages and currents is required to ensure compliance with the low frequency constraints imposed by the DO160 standard. This work does not include the high frequency part EMC of the standard, between $150 \mathrm{kHz}$ and $30 \mathrm{MHz}$.

On the AC side, the goal is to compute the phase-neutral voltage to respect a voltage THD constraint, and to respect the maximum RMS current in the capacitors imposed by the manufacturer datasheet. The PWM signal $V_{P W M}$ is expressed with a double Fourier series [14], leading to equation (1) with Bessel function of first kind $J_{p}$.

$$
V_{P W M}(k \cdot m+p)=\frac{2 \cdot V_{D C}}{\pi \cdot k} \sin \left(\pi \frac{k+p}{2}\right) \cdot J_{p}\left(\pi \frac{k \cdot r}{2}\right)
$$

with $\mathrm{k}$ a positive integer multiple of the switching frequency (Fsw), $\mathrm{p}$ a relative integer for sidebands harmonics, $\mathrm{m}$ modulation index and $r$ amplitude index.

Voltage ripple is reduced with a second order LC filter. AC voltage spectrum $V_{\text {out }}$ is obtained with the transfer function (TF) which depends on the filter and the load, as written in (2).

$$
V_{\text {out }}(f)=T F\left(f, L_{A C}, C_{A C}, R_{\text {load }}, L_{\text {load }}\right) . V_{P W M}(f)
$$

In the case of coupled inductors (Fig 4), it is necessary to evaluate an equivalent filtering inductor. This equivalent value $L_{e q}$ depends on the frequency $f$ and the mutual inductance $M_{A C}$ [15], as expressed in (3).

$$
L_{e q}(f)=2\left(L_{A C}-M_{A C} \cdot \cos \left(\frac{2 \pi f}{N_{\text {interleaved }} m}\right)\right)
$$

With $m=\frac{F_{S w}}{F_{\text {grid }}}$

On the DC side, the following constraints have to be respected:

- Voltage ripple on the DC bus to ensure the hypothesis of a constant DC voltage

- Current ripple in the DC source

- Current ripple in the capacitor for thermal constraint

The developed method takes into account the impact of the AC current ripple on the DC side, and consists in computing the Fourier transform of the current in one transistor, then the summation of the current coming from the 3 phases [16]. The current circulating in the neutral connection between the capacitive DC middle point and the AC capacitor reference point is also taken into account.

\section{Passive component design}

Capacitors are considered with their Equivalent Series Resistor (ESR) to compute the losses, and are constrained with the maximum current given by the manufacturer. Models are based on a continuous interpolation of manufacturer datasheets.

The following paragraphs are dedicated to the sizing models for AC inductors and couplers. The models used for the DC inductor are similar.

Toroidal cores are chosen for AC inductor. Windings are made of Litz wire to decrease copper losses, because of skin and proximity effects. Number and size of Litz strands are optimization parameters. The Dowell method [17] is used to give an analytical formulation of the variation of resistance as a function of the frequency, and is applied to the case of toroidal shapes [18] [19]. Thus, (4) gives the variation of resistance as a function of the frequency and as a function of the number of Dowell layers $\left(N b_{\text {layer }}\right)$. 
$\frac{R_{A C}}{R_{D C}}=x \cdot\left(\frac{\operatorname{sh}(2 x)+\sin (2 x)}{\operatorname{ch}(2 x)-\cos (2 x)}+\frac{2}{3}\left(N b_{\text {layer }}^{2}-1\right) \frac{\operatorname{sh}(x)-\sin (x)}{\operatorname{ch}(x)+\cos (x)}\right)$

with $x=\frac{\frac{\sqrt{\pi}}{2} \Phi_{\text {strand }}}{\Delta}, \Phi_{\text {strand }}$ Litz wire diameter and $\Delta$ the skin depth, which varies with frequency.

For this study, powder core material is chosen for noncoupled inductors. For this material, it is interesting to take into account a variable saturation state of the core. For example, the inductor can saturate during the peak current over a low frequency period, leading to an increased ripple, but with a smaller core area, thus reduced mass. The complete method is given in [20], and consists in computing the magnetic flux ripple with Lenz law and the $\mathrm{B}(\mathrm{H})$ curve of the material. The time-domain variation of flux is then used to compute iron losses with improved Steinmetz equation (iGSE) [21]. Equation (5) is an analytical version of iGSE described in [20] using the switching time of the PWM scheme $t_{\text {off }}$ and $t_{\text {on }}$ function of $\mathrm{j}$ the discretization index from 0 to $\mathrm{m}$. These calculations make it possible to compute also copper losses, and some spectral analysis elements described previously.

$$
\underset{\left.t_{\text {on }}(j)\right)^{1-\alpha}}{P_{\text {ol } i G S E}}=2 k_{i} \Delta B^{(\beta-\alpha)} \frac{1}{T} \sum_{j} \Delta B_{H F}(j)^{\alpha} \cdot\left(t_{o f f}(j)-\right.
$$

with $\alpha$ and $\beta$ the Steinmetz coefficients, and $k_{i}$ coefficient defined in [21].

The design is different for coupled inductors. Nanocrystalline cores are chosen because they are more suitable for this application [22]. This kind of material has very high permeabilities (>20000) and a high saturation flux (1.2T), but also higher prices compared to ferrite cores. The key design element in the sizing of the coupler is the evaluation of the leakage inductor. Many analytical models exist in the literature to evaluate this value. Experiments carried out on different samples revealed that model described in (6) from [22] is very accurate, and will be consequently used for this study.

$$
L_{l k}=\mu_{0} N^{2} \cdot 2\left(D_{\text {ext }}+h\right)\left(\frac{\pi}{6}+0.715 \cos \left(\frac{\theta_{w}}{2}\right)\right)
$$

with $\mu_{0}$ the void permeability, $N$ number of windings, $D_{\text {ext }}$ external core diameter, $h$ core height, and $\theta_{w}$ winding angle $\left(\theta_{w}<180^{\circ}\right)$.

Unlike the inductors, core saturation must be avoided for couplers, because the coupling function would be lost. Consequently, the maximum flux in the core is constrained to the value given by the manufacturer. Core and copper losses are computed in the same way as previously.

\section{E. Differentiability constraint on the models: data interpolation}

A major part of the developed models is innately continuous and differentiable. It is the case for the physical equations, as loss models, or also for the mass estimation because it comes from geometrical definition. However, some parameters are taken from datasheets, and are not continuous, which introduces the notion of relaxation of the real world problem.

The following paragraphs deal with the example of the linear Steinmetz coefficient k variation. The manufacturer [23] only proposes few different relative permeability values for its magnetic cores. Steinmetz coefficient $\mathrm{k}$ varies significantly with the permeability. Then, it is necessary to create a realistic and differentiable model including the three selected points given by the manufacturer (Fig. 7). These considerations prevent from choosing a polynomial interpolation, which moves away from realistic values, or a linear interpolation by parts, which would not be differentiable.

An appropriate way to create this model consists in using transition functions, using for example hyperbolic tangent functions. This kind of function makes it possible to gather smoothly 2 curves. The method is explained in [24]. The goal is to create a transition between $\mathrm{f} 1(\mathrm{x})$ and $\mathrm{f} 2(\mathrm{x})$ as defined by $(7), x_{t}$ being the boundary value.

$$
f(x)= \begin{cases}f_{1}(x) & \text { for } x \leq x_{t} \\ f_{2}(x) & \text { for } x>x_{t}\end{cases}
$$

Transition functions $\mathrm{g} 1(\mathrm{x})$ and $\mathrm{g} 2(\mathrm{x})$, defined in (8), vary respectively from 0 to 1 , and from 1 to $0 . l$ is the transition parameter, which can be adapted depending on the functions (set to 0.2 for Steinmetz coefficient $\mathrm{k}$ ).

$$
\left\{\begin{array}{l}
g_{1}(x)=0.5 *\left(1+\tanh \left(\frac{x_{t}-x}{l}\right)\right) \\
g_{2}(x)=0.5 *\left(1+\tanh \left(\frac{x-x_{t}}{l}\right)\right)
\end{array}\right.
$$

Finally, the global function $\bar{f}$ is computed with (9).

$$
\bar{f}=g_{1} * f_{1}+g_{2} * f_{2}
$$

Fig. 7 places the three points given by the manufacturer and the use of the transition function. In this example, the process is carried out with $f_{1}(\mu)$ and $f_{2}(\mu)$ as linear interpolation and with $\mu=26$ as transition. The blue curve is obtained with only one equation $\bar{f}$, allowing a good differentiation of the model. Transition functions are applied for every Steinmetz coefficient and in other cases where no continuous models are available.

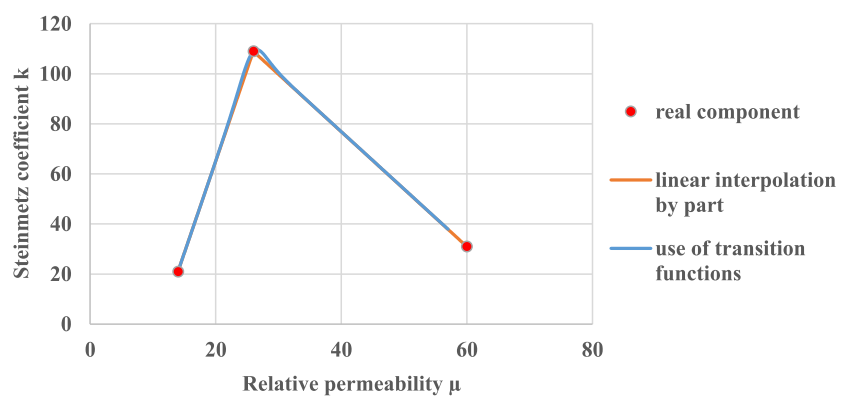

Fig. 7. Steinmetz coefficient $\mathrm{k}$ variation with the relative permeability 


\section{F. Differentiability constraint on the models: Non integer interleaving level}

Allowing a continuous variation of the interleaving level introduces non-realistic results in some models. The typical example is the computation of the AC spectrum with Bessel functions described in (1). This model is only valid for integer value of interleaving levels.

This issue is solved by creating an interpolation between the two surrounding integers, at each iteration during the optimization process. For example, if the interleaving value chosen by the algorithm is 2.416 , the model is computed twice, with $\mathrm{N}_{-}$interleaved $=2$ and $\mathrm{N}_{-}$interleaved $=3$. The output value is then obtained with a linear interpolation.

This method makes it possible to get a continuous value of THD function of the interleaving level, as shown in Fig. 8 (the same approach is used for individual harmonic level). However, the model is not fully differentiable. There is a break in the derivative at the integer interleaving level values, because the values used for the interpolation change. In this case, the issue is not so problematic for the algorithm because the change in the derivative is not so important, as illustrated in Fig. 8, while it was very disturbing for Steinmetz coefficient k in Fig. 7.

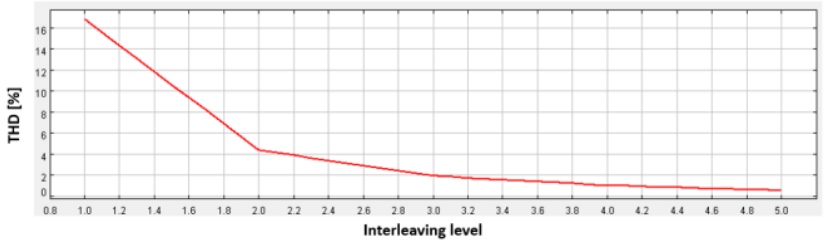

Fig. 8. AC Voltage THD function of the interleaving level

\section{OPTIMIZATION RESULTS}

\section{A. Optimization problem introduction}

A gradient-based optimization algorithm, using the Sequential Quadratic Programming method (SQP) [25], is used with the framework CADES [26] [27]. Standard ML (SML) programming language is used because it is an appropriate language to compute the derivatives. For more complex models, C++ language is used with automatic code differentiation library [28].

Fig. 9 shows the main links between the variables and the models developed previously, with the equation references of each model introduced in the previous section. The initial conditions of the input parameters are selected manually, with the designer experience. It is shown in section IV.A that it has no impact on the result.

The input variables appear in blue and can vary during the solving. The constrained output variables are in green. The objective function is the overall mass, which is the sum of the mass of the different components. Losses are used to compute the thermal constraint of each component, as well as to compute the global efficiency. Even if this figure is displayed with blocks referring to the different sub-systems, the algorithm performs a global solving, computing every output variable at the same time. The models, represented by the white boxes, are not fully explained, as it is not the aim of this article. Thus, the designers can use their own model without limitation of complexity, which will be more suitable for his application. Models communicate between each other, and their outputs are linked by global variables. Fig. 9 illustrates the necessity to adopt a global approach considering the multiple links between the input variables and the constrained ones.

\section{B. Detailed results on the selected application}

This section deals with the global inverter design (DC filter, AC filter, semi-conductors), with the constraints listed in Table I, and a nominal power of $10 \mathrm{kVA}$.

Results are shown as Mass-Efficiency Pareto fronts, which are the non-dominated solutions of the space. Fig. 10 shows a Pareto front at $10 \mathrm{kVA}$, with an interleaving level $\mathrm{N}$ which can vary continuously between 1 and 5. In Fig. 11, solutions using $\mathrm{N}=1$ and $\mathrm{N}=2$ are superposed to highlight this continuous variation.

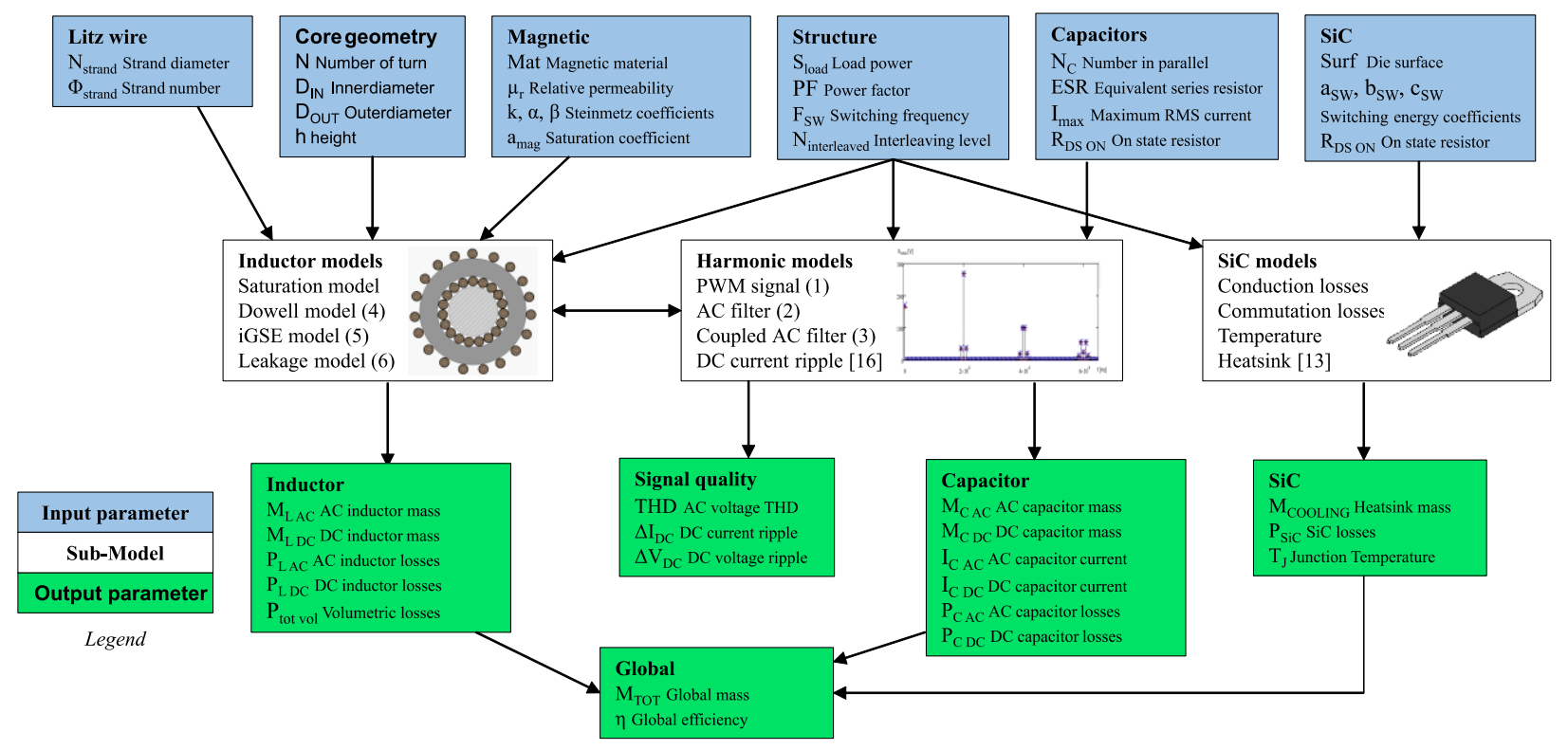

Fig. 9. Global schematic of the optimization problem 
When the efficiency constraint is not so strong $(<97.5 \%)$, the optimal topology is a non-interleaved inverter. If the required efficiency is higher than $97.5 \%$, then the best solution consists in interleaving two inverters. Many constraints are active in this design, but with unbalanced impact. Sensitivity analyses in section IV allows understanding which variables have the most important impact on the converter.

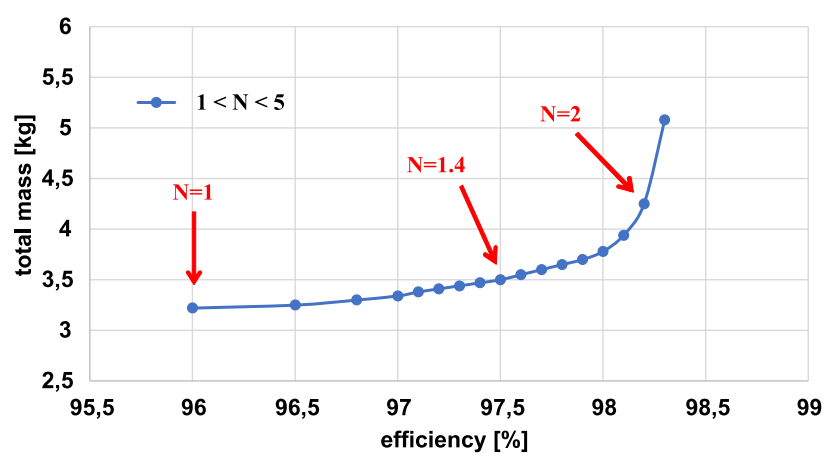

Fig. 10. Pareto front Mass-Efficiency with a variable interleaving level

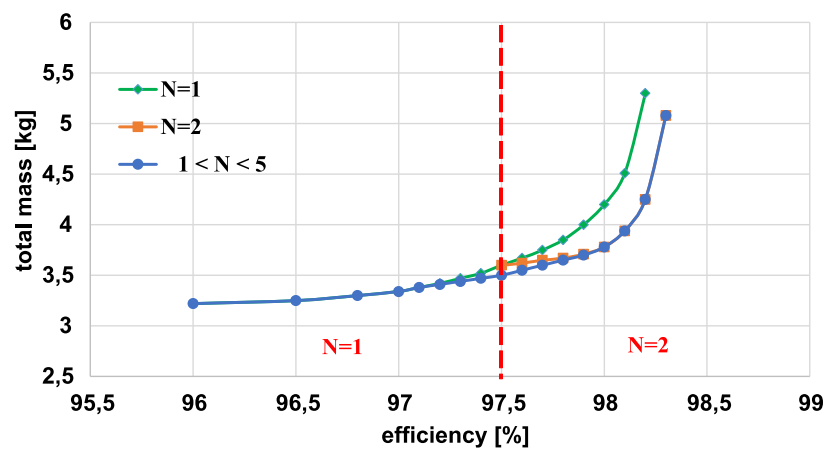

Fig. 11. Pareto front Mass-Efficiency with fixed interleaving levels

This result shows that using a relaxation of the real solution space, even on very crucial variables as the interleaving level, gives results close to the real world designs. In this example, at $97.5 \%$, the optimal mass obtained for $\mathrm{N}=1.4$ increases of only $2 \%$ to find a solution with an integer interleaving level. A study about the impact of the relaxation of the real world problem is developed in paragraph IV.C.

Optimization is then carried out for the inverter structures with magnetic couplers in cyclic cascade. Results are shown in Fig. 12, and are compared to the non-coupled solutions. With these new solutions, if the required efficiency is higher than $97.3 \%$, then the optimal topology becomes two interleaved inverters with magnetic couplers. Below this efficiency limit, the classic inverter remains the best choice. Coupling inductors becomes attractive for high efficiency because core losses are reduced compared to the classical case. For lower efficiency, the non-interleaving solution is lighter because there is no need to double the number and mass of some components compared to the interleaved or coupled solution.

Fig. 13 and 14 show the detailed inverter design, for the three structures with $98 \%$ efficiency. It is worth noting that the AC magnetic component, inductor or coupler, is the most important one in terms of mass and loss. The losses in the active devices are also a key design parameter. These considerations justify the modeling effort on these components.

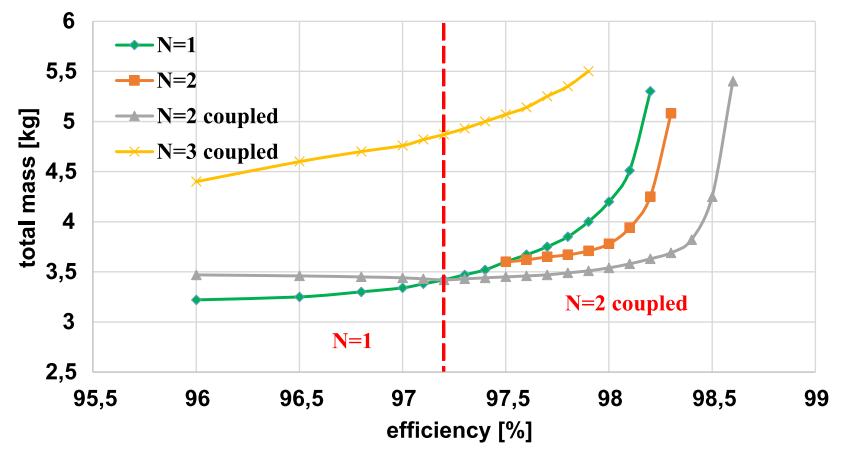

Fig. 12. Pareto front Mass-Efficiency non-coupled and coupled inductors at 10 kVA
Reference

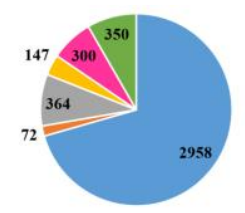

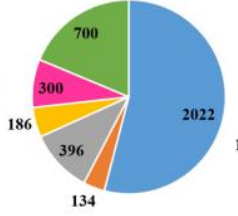

Interleaved

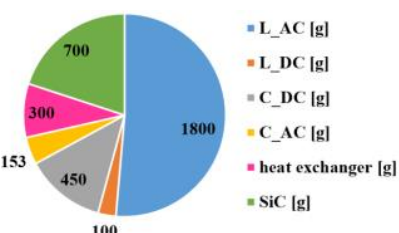

Coupled
Fig. 13. Optimal distribution of the mass for $98 \%$ efficiency at $10 \mathrm{kVA}$

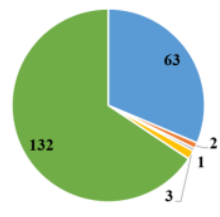

Reference

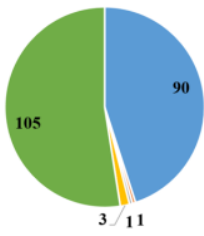

Interleaved

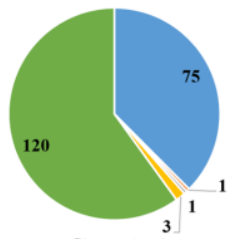

Coupled
Fig. 14. Optimal distribution of the losses for $98 \%$ efficiency at $10 \mathrm{kVA}$

Finally, when the efficiency constraint increases, the algorithm chooses to decrease the switching frequency to reduce the switching losses. Increasing the frequency makes the filter design more difficult, which leads to an increase of the filter mass. These charts help the designer to understand the key design parameters and consequently how to determine which components require additional modeling effort.

A Pareto front at $100 \mathrm{kVA}$ is presented on Fig. 15. The coupled topology remains the best solution for high efficiency constraints. However, the non-interleaved solution is now dominated by the two interleaved inverters topology. In conclusion, when the converted power increases, it is worth to choose solutions with higher interleaving level, because it becomes relevant to share the currents between the different legs.

In this example, 10 minutes is an average solving time to build a Pareto front composed of 10 optimal points (for 10 different efficiency targets). The average number of iteration is 120, divided in 30 iterations for the first point (with random initial conditions), and then 10 iterations for the 9 remaining points. Solving the 9 remaining points is easier as the algorithm keeps in mind the last optimal point, which is close as there is only a small shift in the efficiency target. It is worth noting that this solving time could be decreased further. In fact, a detailed model for DC link harmonics was chosen. Computing and 
differentiation of this model consumes approximately $90 \%$ of the time at each step. With a simplified model, a Pareto front could be drawn in less than 1 minute.

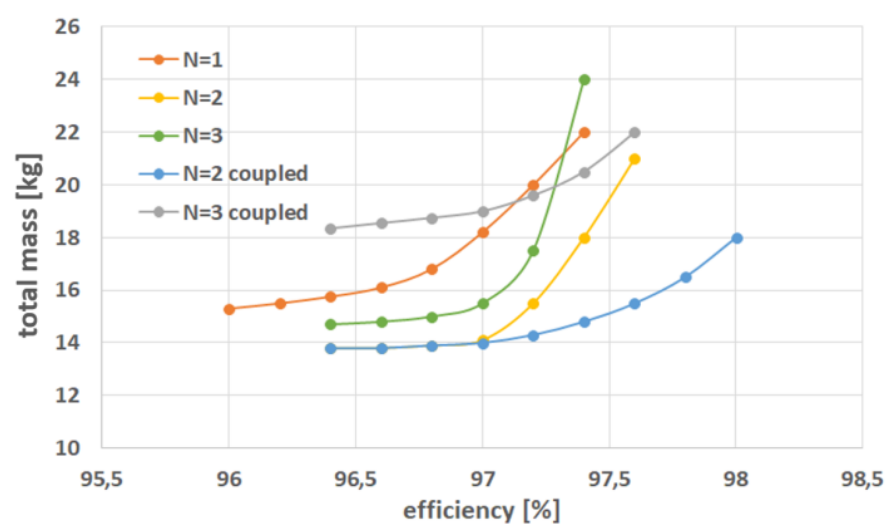

Fig. 15. Pareto front Mass-Efficiency at $100 \mathrm{kVA}$

\section{Methodology VAlidation}

\section{A. Global optimality}

The major drawback that can be encountered with a gradientbased algorithm is a convergence to a local optimum, instead of a global optimum. This issue can arise if the solution space is not convex. In this case, the result depends on the initial conditions.

As it is impossible to prove the optimality of the solution, the following experiment is proposed to discuss about this topic, involving a multi-start process on sensitive input parameters. 10 optimizations are carried out for 10 different initial switching frequencies, from $10 \mathrm{kHz}$ to $100 \mathrm{kHz}$. The 10 results found by the algorithm are identical. Only the solving time varies function of the initial point: from 93 seconds up to 154 seconds. This experiment has been carried out for many input variables (relative permeability, number of winding, core diameter ...). The 40 optimizations carried out with different initial parameters converge towards the same result.

For each modification of an initial parameter value, the whole path followed by the algorithm changes. In other words, the evolution of the selected parameter changes as well as the evolution of other parameters. It means that the algorithm explores a different solution space each time compared to the initial case, which is the requested behaviour to investigate the global optimality of the solution.

Even if the problem is not fully convex, the algorithm always finds the global solution of the problem during this particular experiment. This good behavior of the tool is possible because of the automatic code differentiation implemented in the CADES framework by [27], and because the models used are mainly written with convex functions. In addition, the work introduced in parts II.E and II.F about how to deal with noncontinuous models make it possible to ensure the convergence of the algorithm. If the designer wonders whether it is a global solution or not, which is still possible because the solution space is never fully convex, the multi-start process is still available to remove any doubt, however increasing the design time.

\section{B. Sensitivity analysis}

The goal of this section is to study the impact of the variation of the input parameters on the objective function. The proposed method consists in carrying optimizations with a fixed input parameter, close to the optimal parameters previously obtained. Consequently, losses and efficiency values are identical to the reference case as the algorithm always finds a way to target the constraint limit. This experiment will restrain the space, and the new solutions should be consequently sub-optimal.

In Fig. 16, a shift of +/- 10\% have been imposed on some key input variables, compared to an optimal point obtained previously with the specifications given in Tables I and II ( $\mathrm{S}_{\text {load }}$ $=10 \mathrm{kVA}$ and $\eta>97 \%$ ). For example, when the interleaving level is set to $+10 \%$ or $-10 \%$ compared to the optimal solution, with the other input still free to vary during the optimization, the computed mass of the converter is $2 \%$ heavier.

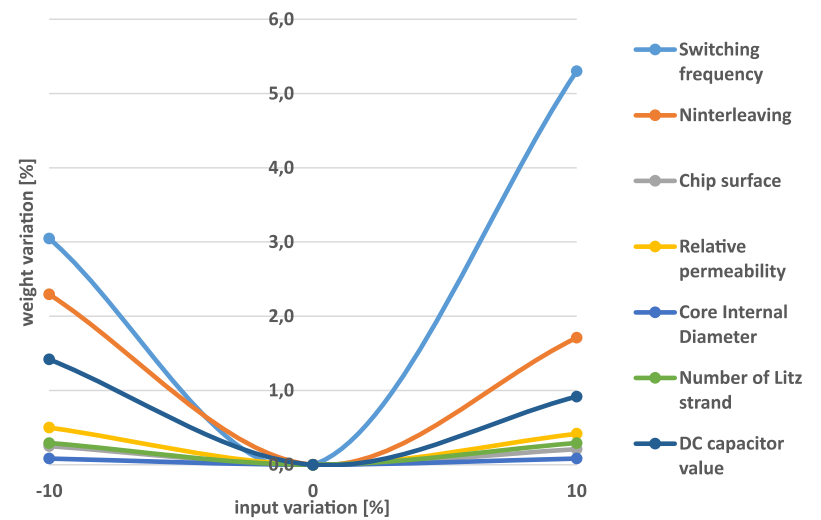

Fig. 16. Global mass variation function of a shift of input parameter

The first conclusion of this study is about the relative impact of each input parameter on the result. It is worth noting that the switching frequency and the interleaving level have more impact than the other variables. This is not surprising considering that these variables influence every sub-system of the converter. With this study, the designer should be able to understand which choices have the greatest impact in the design.

The second conclusion is about the robustness of the design. For example, choosing a core permeability different from the optimal one (for practical reasons, or since the real permeability often varies from the targeted one $-20 \%$ variation is commonly encountered-) is not a serious issue. Shifting an input parameter of $10 \%$ induces a small change in the global mass of the system. This study reveals that the algorithm has found a solution in a "flat" part of the design space, which is a proof of robustness. Therefore, it is possible to adapt the design to find performances close to the optimal point, even if one of the input parameters changes.

\section{Impact of the relaxation}

Working in a design space with relaxation on some variables is not a conventional approach in the design of power converters. The questions raised by this method can tackle the trust on the results obtained in this particular space, compared to the real ones. Another concern can be about the methodology 
to design a real converter, even if it is not the main goal of the developed optimization tool, which should be used in a predesign step.

The method to go back to the real world consists in assigning one by one the input variables to real values, which can be integers data or given by the manufacturer. Between each assignment, an optimization is carried out to adapt the other input variables. The remaining question is about the choice of assignment order of the variables. Assigning parameter $\mathrm{X}$ first can shift the solution to another solution space, which could be different if parameter $\mathrm{Y}$ had been chosen first.

In the studied case, there are 11 relevant input parameters. Considering Fig. 13 and 14, the most important parameters are the AC inductor and the semi-conductors. Thus, the first steps in the process focus on the assignment of these 2 parts. Fig. 17 shows the four first steps of the process (and the initial point), with four different order of assignment. The four parameters are: area of semi-conductor chip, diameters of the toroid, height of the core, and number of strand of Litz wire.

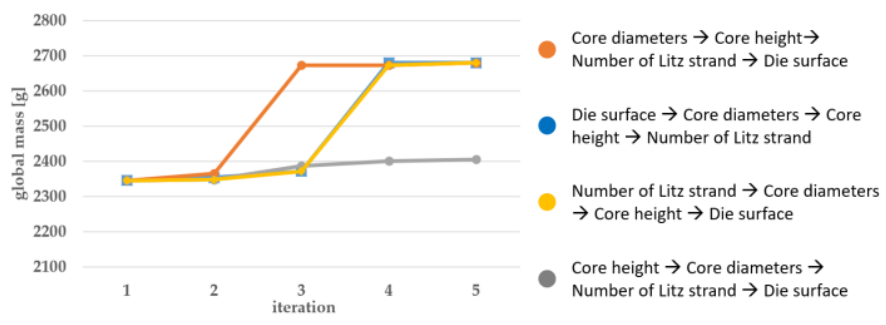

Fig. 17. Different paths to go back to the real world

The grey path gives better result compared to the orange, yellow and blue paths. The designer has to choose the good path with his experience. During this step, the role of the power electronics designer becomes dominating. The designer can fix some technological dependent parameters, and use the tool to evaluate the divergence of the design, compared to the optimal solution. A sensitivity analysis on the objective function, as shown in Fig. 16, is not sufficient to choose the best order because the path has also an impact on the constraints. This example illustrates the necessary expertise to go back to a real converter with this method. However, the main conclusions obtained in the space with relaxation on the global optimization and the comparison of topologies are still valid and very interesting, what is the main goal of the proposed methodology.

The process has been carried out for different points over a Pareto curve. Fig. 18 compares the solutions from the optimization tool and the solutions after the main step of going back to the real world. The shape of the curves remains identical, with an increase of the global mass in the real world compared to the optimization world, which is normal because the real space is smaller due to the relaxation. This increase does not exceed $14 \%$, which is very acceptable to compare different candidate solutions, as different structures in power electronics. Using a relaxation of the real world space is thus suitable for a pre-design optimization tool.

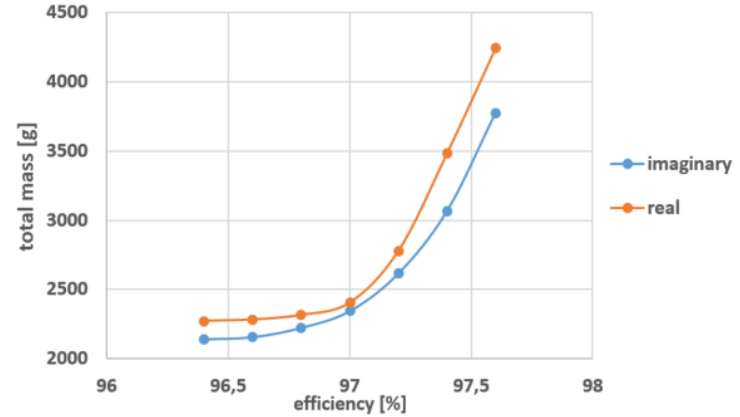

Fig. 18. Difference between optimization solutions and manufacturable solutions at $10 \mathrm{kVA}$

As the proposed process is not fully satisfying, for example on the choice of assignment order, a combinatory algorithm could be proposed in the future (branch and bounds [29] for example), for this re-discretization step. This kind of algorithms requires time for solving, but it is not an issue in this case as the solution space has been reduced by the gradient-based optimization.

\section{EXPERIMENTAL VALIDATIONS}

A 10kW prototype (Fig. 19) has been built in order to validate the models discussed in section II, and the optimization results introduced in section III. It is composed of 6 phases legs, which can be used to build a non-interleaved, an interleaved or a coupled two levels 3 phases inverter. Calorimetric test benches (Fig. 20) are used to measure precisely the losses of active and passive components [20].

Tables III compares the results obtained with the optimization tool to the results measured in experiment, for the non-interleaved, interleaved and coupled cases.

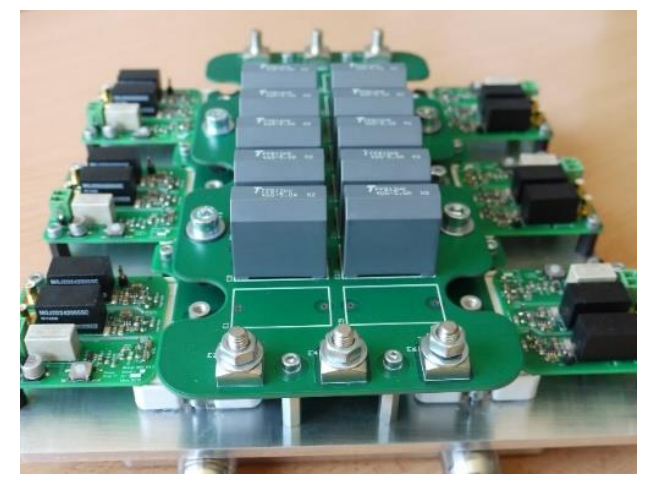

Fig. 19. 3-Phase $10 \mathrm{kVA}$ inverter developed

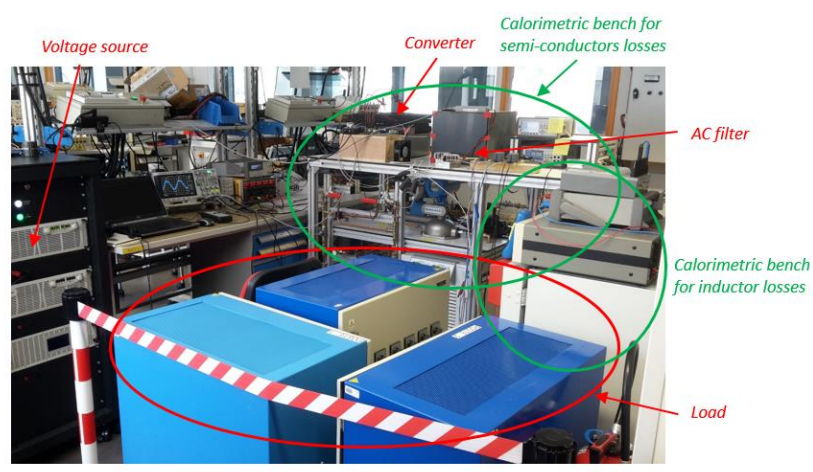

Fig. 20. Experimental setup with the test benches 
TABLE III

COMPARISON BETWEEN OPTIMIZATION RESULTS AND EXPERIMENTS FOR THE THREE DEVELOPED SOLUTIONS

\begin{tabular}{|c|c|c|c|c|c|c|c|c|c|c|}
\hline & & \multicolumn{3}{|c|}{ Non-interleaved } & \multicolumn{3}{|c|}{ Interleaved } & \multicolumn{3}{|c|}{ Coupled } \\
\hline Quantity & Unit & Design & Experiment & Error $[\%]$ & Design & Experiment & Error $[\%]$ & Design & Experiment & Error $[\%]$ \\
\hline AC inductor losses & $\mathrm{W}$ & 29 & 28 & 3.4 & 17.8 & 16.9 & 5.1 & 21.3 & 21.8 & 2.3 \\
\hline Semi-conductor losses & $\mathrm{W}$ & 130 & 123 & 5.4 & 111 & 99 & 11 & 153 & 145 & 4.5 \\
\hline Global losses & $\mathrm{W}$ & 220 & 219 & 0.5 & 220 & 213 & 3.6 & 220 & 227 & 3.0 \\
\hline RMS AC capacitor current & A & 5.6 & 5.4 & 3.5 & 2.5 & 3 & 20 & 9.1 & 9 & 1.1 \\
\hline RMS DC capacitor current & A & 21.9 & 22 & 0.5 & 9.1 & 9 & 1.1 & 11.5 & 18 & 56 \\
\hline DC source current ripple & $\mathrm{A}$ & 0.9 & 0.6 & 33 & 0.7 & 0.6 & 14 & 0.93 & 1 & 7.5 \\
\hline DC source voltage ripple & $\mathrm{V}$ & 5 & 4 & 20 & 4.6 & 5 & 8.7 & 1.4 & 3 & 114 \\
\hline AC THD on voltage & $\%$ & 2.1 & 1.8 & 14 & 1.1 & 1.3 & 18 & 2 & 2 & 0 \\
\hline AC max voltage harmonics & $\%$ & 2.0 & 1.7 & 15 & 0.74 & 0.66 & 11 & 1.3 & 1.1 & 15 \\
\hline
\end{tabular}

The results show few differences between the optimization results and the measured values. This proves the good accuracy of the developed analytical models, which are required to build pre-design optimization tools in power electronics with gradient-based algorithms.

The only issue revealed by the experiments, and confirmed by simulation, is the calculation of the RMS current in the DC capacitors in the coupled case. This is due to a lack of consideration of the AC ripple on the DC side. This error is also reported on the DC bus voltage ripple. However, the design of the DC bus capacitor is not the most important point in this case, and this modeling issue does not modify the conclusions.

\section{CONCLUSION}

This paper introduced an optimization method, using a gradient-based algorithm, to pre-design power converters in a highly constrained environment. The developed tool is able to help the designer to make important choices during the first steps of a project.

The chosen method can be seen as a fair compromise between convergence speed and modelling accuracy. Compared to another method in the deterministic algorithm family [7], models can be written with more freedom with the proposed approach, which allows using models that are more detailed. However, this method do not ensure at $100 \%$ the global optimality of the solution, which is one of the main benefits of [7], in addition to the efficiency of the convex solvers to carry out an optimization in few seconds.

On the other hand, gradient-based methods can explore a wider solution space in a reasonable time compared to stochastic method, but need a relaxation step to build a real converter. Thereby, the developed methodology can be selected as of one of the design automation tools required to improve the complete design process of power converters.

Analytical models, continuous and differentiable, dedicated to the design of voltage inverters, have been developed and integrated in the optimization process. These models address both active and passive component, and also spectral analysis, for different interleaved structures. Some examples show how to adapt the models to be compliant with the gradient-based method.

The developed tool is used in this work to compare different topologies in the same conditions, which helps the designer to make the first choices in the pre-design steps of the project. The optimal topology depends mainly on the power level and the required efficiency of the converter.

The validity of the results using a relaxation of the real world problem has been proved by sensibility study and experimental results. Working in this particular space does not affect significantly the analysis on the result. The design tool is able to compute quickly and accurately the global optimum of the problem. The accuracy of analytical models is also proved with experimental measurements.

\section{REFERENCES}

[1] B. Sarlioglu and C. T. Morris, 'More Electric Aircraft: Review, Challenges, and Opportunities for Commercial Transport Aircraft', IEEE Trans. Transp. Electrification, vol. 1, no. 1, pp. 54-64, Jun. 2015, doi: 10.1109/TTE.2015.2426499.

[2] I. Laird, X. Yuan, J. Scoltock, and A. J. Forsyth, 'A Design Optimization Tool for Maximizing the Power Density of 3-Phase DCAC Converters Using Silicon Carbide (SiC) Devices', IEEE Trans. Power Electron., vol. 33, no. 4, pp. 2913-2932, Apr. 2018, doi: 10.1109/TPEL.2017.2705805.

[3] K. Park, R. Kieferndorf, U. Drofenik, S. Pettersson, and F. Canales, 'Optimization of LCL filter with Integrated Intercell Transformer for Two-Interleaved High Power Grid-Tied Converters', IEEE Trans. Power Electron., pp. 1-1, 2019, doi: 10.1109/TPEL.2019.2926312.

[4] D. O. Boillat, F. Krismer, and J. W. Kolar, 'Design Space Analysis and $\rho-\eta$ Pareto Optimization of LC Output Filters for Switch-Mode AC Power Sources', IEEE Trans. Power Electron., vol. 30, no. 12, pp. 6906-6923, Dec. 2015, doi: 10.1109/TPEL.2015.2393151.

[5] Y. Shen, S. Song, H. Wang, and F. Blaabjerg, 'Cost-VolumeReliability Pareto Optimization of a Photovoltaic Microinverter', in 2019 IEEE Applied Power Electronics Conference and Exposition (APEC), Mar. 2019, pp. 139-146, doi: 10.1109/APEC.2019.8722043.

[6] M. Mirjafari, S. Harb, and R. S. Balog, 'Multiobjective Optimization and Topology Selection for a Module-Integrated Inverter', IEEE Trans. Power Electron., vol. 30, no. 8, pp. 4219-4231, Aug. 2015, doi: 10.1109/TPEL.2014.2353055.

[7] A. Stupar, T. McRae, N. Vukadinović, A. Prodić, and J. A. Taylor, 'Multi-Objective Optimization of Multi-Level DC-DC Converters Using Geometric Programming', IEEE Trans. Power Electron., vol. 34, no. 12, pp. 11912-11939, Dec. 2019, doi: 10.1109/TPEL.2019.2908826.

[8] M. Delhommais, J. Schanen, F. Wurtz, C. Rigaud, and S. Chardon, 'First order design by optimization method: Application to an interleaved buck converter and validation', in 2018 IEEE Applied Power Electronics Conference and Exposition (APEC), Mar. 2018, pp. 944-951, doi: 10.1109/APEC.2018.8341128.

[9] M. Mirjafari and R. S. Balog, 'Survey of modelling techniques used in optimisation of power electronic components', IET Power Electron., vol. 7, no. 5, pp. 1192-1203, May 2014, doi: 10.1049/ietpel.2013.0321.

[10] E. Laboure, A. Cuniere, T. A. Meynard, F. Forest, and E. Sarraute, 'A Theoretical Approach to InterCell Transformers, Application to 
Interleaved Converters', IEEE Trans. Power Electron., vol. 23, no. 1, pp. 464-474, Jan. 2008, doi: 10.1109/TPEL.2007.911786.

[11] Microsemi, 'APTMC120AM55CT1AG'.

https://www.microsemi.com/existing-

parts/parts/112756?catid=1349\#resources (accessed Mar. 24, 2020).

[12] A. Acquaviva and T. Thiringer, 'Energy efficiency of a SiC MOSFET propulsion inverter accounting for the MOSFET's reverse conduction and the blanking time', in 2017 19th European Conference on Power Electronics and Applications (EPE'17 ECCE Europe), Sep. 2017, p. P.1-P.9, doi: 10.23919/EPE17ECCEEurope.2017.8099052.

[13] Y. Fefermann et al., 'Hybrid-Electric Motive Power Systems for Commuter Transport Applications', Sep. 2016.

[14] D. G. Holmes and T. A. Lipo, 'Modulation of ThreePhase Voltage Source Inverters', in Pulse Width Modulation for Power Converters: Principles and Practice, IEEE, 2003, pp. 215-258.

[15] N. Bouhalli, 'Muticell parallel interleaved coupled converters: analysis and integration', PhD, Institut National Polytechnique de Toulouse - INPT, 2009.

[16] A. Voldoire, J.-L. Schanen, J.-P. Ferrieux, C. Gautier, and C. Saber, 'Analytical Calculation of DC-Link Current for N-Interleaved 3-Phase PWM Inverters Considering AC Current Ripple', in 201921 st European Conference on Power Electronics and Applications (EPE '19 ECCE Europe), Sep. 2019, p. P.1-P.10, doi: 10.23919/EPE.2019.8915183.

[17] P. L. Dowell, 'Effects of eddy currents in transformer windings', Proc. Inst. Electr. Eng., vol. 113, no. 8, pp. 1387-1394, Aug. 1966, doi: 10.1049/piee.1966.0236.

[18] G. Lefevre, H. Chazal, J. P. Ferrieux, and J. Roudet, 'Application of Dovvell method for nanocrystalline toroid high frequency transformers', in 2004 IEEE 35th Annual Power Electronics Specialists Conference (IEEE Cat. No.04CH37551), Jun. 2004, vol. 2, pp. 899-904 Vol.2, doi: 10.1109/PESC.2004.1355538.

[19] J. A. Ferreira, 'Improved analytical modeling of conductive losses in magnetic components', IEEE Trans. Power Electron., vol. 9, no. 1, pp. 127-131, Jan. 1994, doi: 10.1109/63.285503.

[20] A. Voldoire, J.-P. Ferrieux, J.-L. Schanen, C. Rizet, C. Gautier, and C. Saber, 'Validation of Inductor Analytical Loss Models under Saturation Conditions for PWM inverter', in 201921 st European Conference on Power Electronics and Applications (EPE '19 ECCE Europe), Sep. 2019, p. P.1-P.9, doi: 10.23919/EPE.2019.8915174.

[21] K. Venkatachalam, C. R. Sullivan, T. Abdallah, and H. Tacca, 'Accurate prediction of ferrite core loss with nonsinusoidal waveforms using only Steinmetz parameters', in 2002 IEEE Workshop on Computers in Power Electronics, 2002. Proceedings., Jun. 2002, pp. 36-41, doi: 10.1109/CIPE.2002.1196712.

[22] F. Forest, E. Labouré, T. A. Meynard, and V. Smet, 'Design and Comparison of Inductors and Intercell Transformers for Filtering of PWM Inverter Output', IEEE Trans. Power Electron., vol. 24, no. 3, pp. 812-821, Mar. 2009, doi: 10.1109/TPEL.2008.2007900.

[23] Magnetics, 'Magnetics Powder Core Catalog'. [Online]. Available: https://www.mag-inc.com/Media/Magnetics/FileLibrary/Product\%20Literature/Powder\%20Core\%20Literature/2017Magnetics-Powder-Core-Catalog.pdf.

[24] L. D. Roper, 'The Hyperbolic Tangent World', Apr. 2019. http://www.roperld.com/science/Mathematics/HyperbolicTangentWor ld.htm (accessed Jan. 28, 2020).

[25] P. T. Boggs and J. W. Tolle, 'Sequential Quadratic Programming', Acta Numer., vol. 4, pp. 1-51, Jan. 1995, doi: 10.1017/S0962492900002518.

[26] B. Delinchant, L. Estrabaud, L.Gerbaud and F. Wurtz (2013). MultiCriteria Design and Optimization Tools. Integrated Design by Optimization of Electrical Energy Systems. 193-245. doi: 10.1002/9781118561812.ch5.

[27] B. Delinchant, D. Duret, L. Estrabaut, L. Gerbaud et al.. (2007). An optimizer using the software component paradigm for the optimization of engineering systems. COMPEL: The International Journal for Computation and Mathematics in Electrical and Electronic Engineering. 26. 368-379. doi: 10.1108/03321640710727728.

[28] P. Enciu, L. Gerbaud, and F. Wurtz, 'Automatic Differentiation for Sensitivity Calculation in Electromagnetism: Application for Optimization of a Linear Actuator', IEEE Trans. Magn., vol. 47, no. 5, pp. 1238-1241, May 2011, doi: 10.1109/TMAG.2010.2073452.

[29] C. Roucairol, 'Parallel branch and bound algorithms- an overview', INRIA, RR-0962, Jan. 1989. Accessed: Jun. 26, 2020. [Online]. Available: https://hal.inria.fr/inria-00075597. 